\title{
Riqueza total de especies de plantas vasculares en un bosque andino de la Cordillera central de Colombia
}

\author{
Heriberto David-Higuita ${ }^{1}$ \& Esteban Álvarez-Dávila ${ }^{2,3 *}$ \\ 1. Universidad de Antioquia, Herbario HUA, Calle 67 Número 53 - 108, Medellín, Antioquia, Colombia; \\ heridavidhi@yahoo.com \\ 2. Escuela ECAPMA - Universidad Nacional Abierta y a Distancia (Colombia), Calle 14S \#23 - 14, Bogotá, Colombia. \\ 3. Fundación ConVida, FCV, Cra. 48 \#20114, Medellín, Antioquia, Colombia; esalvarez3000@gmail.com \\ * Correspondencia.
}

Recibido 13-VI-2017. Corregido 18-X-2017. Aceptado 17-XI-2017.

\begin{abstract}
Total richness of vascular plant species in an Andean forest of the Central Cordillera, Colombia. Studies of plant diversity in tropical forests are usually restricted to trees or other groups of woody plants above a certain stem diameter. However, surveys that include all forms of live plants with no restrictions on their sizes, clearly indicate that non-woody plants are equally important. In this study, we reported the total species richness of vascular plants species (TSR) in one hectare plot in an Andean forest in Northwestern Colombia $\left(6^{\circ} 12^{\prime} 48^{\prime \prime} \mathrm{N}\right.$ $\& 75^{\circ} 2932^{\prime \prime W}$ ). We evaluated the relative contribution of the different growth habits and the effect of the plant size, to TSR. We measured all individuals with diameter (D) $\geq 5 \mathrm{~cm}$ in the hectare and all the vascular plants of all sizes, including epiphytes, in a subsample of 0.25 ha. A total of 14545 individuals distributed in 318 species, 72 families (considering Pteridophyta as one group) and 171 genera were registered. Most of the species showed a (D) $<10 \mathrm{~cm}(99.7 \%)$ and $<2.5 \mathrm{~cm}(94.4 \%)$. The no-arboreal species (ground herbs, epiphytes and vines) represented $54.3 \%$ of the total species reported in the plot, indicating that they are important in the structure, composition and species richness of this montane forest. Our results coincide with similar studies in other tropical forests. We concluded that to get a more detailed knowledge of the floristic diversity of a site, it is advisable to: 1) amplify the size range of the plants generally considered in the floristic inventories and 2) to include nonwoody species. This information is crucial for making better decisions in local and global conservation efforts. Rev. Biol. Trop. 66(1): 227-236. Epub 2018 March 01.
\end{abstract}

Key words: floristic diversity; total count; growth habits; non-woody species; measuring ranges.

Los reportes de la diversidad de especies de plantas en los bosques tropicales se basan tradicionalmente en inventarios de vegetación en parcelas de área conocida y restringidos a ciertos subgrupos, usualmente árboles con diámetro del tronco $(\mathrm{D}) \geq 10 \mathrm{~cm}$ (ter Steege et al., 2013) o plantas leñosas con (D) $\geq 2.5$ cm (Qian \& Song, 2013). Si bien este tipo de inventarios han sido útiles para ilustrar la gran riqueza de plantas de los bosques tropicales, así como para entender los patrones espaciales y sus determinantes ambientales, es necesario reconocer sus limitaciones. En particular, estos inventarios excluyen componentes importantes de la flora como epífitas, lianas, hierbas terrestres, arbustos y además los individuos juveniles de especies arbóreas, que no han alcanzado el diámetro mínimo definido, que en conjunto pueden representar hasta el $70 \%$ del total de las especies en un área, como lo demuestran algunos pocos estudios realizados en Ecuador, Guayanas, Colombia, Costa Rica, Filipinas y Bolivia (Linares-Palomino et al., 2009). Por tanto, es posible afirmar que existe un gran vacío de conocimiento sobre la diversidad de la flora tropical lo cual obstaculiza la investigación ecológica y su aplicación a los programas de conservación a diferentes escalas (Prance, 
Beentje, Dransfield, \& Johns, 2000; Phillips et al., 2003; Mora, Tittensor, Adl, Simpson, \& Worm, 2011).

En este trabajo reportamos el número total de especies de plantas vasculares en un bosque del norte de los Andes con base en una parcela de una hectárea; se incluyen todos los hábitos de crecimiento presentes en el bosque (árboles, arbustos, hierbas terrestres, epífitas y escandentes), considerando los individuos con una altura mínima igual a $20 \mathrm{~cm}$. Adicionalmente, se compiló una base de datos con los pocos estudios publicados que reportan información de la riqueza total en parcelas, con el objetivo de comparar los resultados con otros bosques neotropicales. Este estudio representaría el primer reporte de conteo total de especies de plantas vasculares para un bosque andino en Colombia. Para esta investigación, quisimos responder a las siguientes preguntas: ¿cuál es el número total de especies de plantas vasculares?, ¿cuál es la contribución de las especies no arbóreas a la riqueza total de especies?, y ¿cuál es el efecto de reducir el diámetro mínimo de los individuos incluidos en el inventario?

\section{MATERIALES Y MÉTODOS}

Área de estudio: El estudio se realizó en la Reserva Natural Montevivo (RNM) $\left(6^{\circ} 12^{\prime} 48^{\prime \prime} \mathrm{N}-75^{\circ} 29^{\prime} 32^{\prime \prime} \mathrm{W}\right)$, localizada en la cordillera central, a $15 \mathrm{~km}$ al oriente de la ciudad de Medellín, Colombia. El sitio tiene un altitud entre 2500 - $2680 \mathrm{msnm}$, y una precipitación de $2500 \mathrm{~mm} /$ año, y se ubica en la zona de vida Bosque muy húmedo Montano Bajo (sensu Holdridge, Grenke, Hatheway, Liang, \& Tosi, 1971). El relieve de la parcela está formado de pequeñas colinas con pendientes entre $25-45^{\circ}$. Sus suelos son ácidos, de textura arcillosa, con cenizas volcánicas tipo andisol, con alto contenido de materia orgánica (David-Higuita \& Álvarez-Dávila, 2015).

En el 2003 se estableció una parcela permanente de una hectárea $(100 \times 100 \mathrm{~m})$ subdividida en cuadrantes de $20 \times 20 \mathrm{~m}^{2}$ con el objetivo de cuantificar la riqueza total de especies de plantas vasculares (RTE de aquí en adelante). El $50 \%$ del área de la parcela correspondió a un bosque maduro en buen estado de conservación, mientras que en el restante $50 \%$, fue sujeto de extracción selectiva de madera hace décadas. Luego del establecimiento de la Reserva en 1977 (hace 40 años), el bosque se encuentra protegido y no existen evidencias de disturbios recientes. Dentro de la parcela se identificaron y contaron todas las especies o morfoespecies (cuando la identificación completa no fue posible) presentes, agrupándolas por hábitos de crecimiento; la mayor parte del trabajo se realizó durante la época de menor precipitación entre enero y marzo del 2003. Se definieron seis hábitos de crecimiento usados comúnmente en estos estudios (Duivenvoorden, 1994; Londoño \& Álvarez, 1997; Galeano, Suárez, \& Balslev, 1998; Linares-Palomino et al., 2009) árboles, escandentes herbáceos o enredaderas, escandentes leñosos o lianas, hierbas terrestres y epífitas. Para una descripción más detallada de las características de cada hábito consultar el Apéndice digital 1.

Se construyeron curvas de rarefacción área/riqueza para especies, géneros y familias con base en el número total de cada taxa registrado en los cuadrantes de $20 \times 20 \mathrm{~m}^{2}$, y se estimó el número máximo esperado usando el modelo Chao 2, como una medida de la eficiencia o completitud del muestreo (Colwell, 2013). La curva de rarefacción de especies se calculó con el método propuesto por Colwell et al. (2012), que permite extrapolar los datos máximo hasta tres veces el área de muestreo, y así poder comparar los resultados con otros estudios para el neotrópico, que tienen diferentes tamaños de muestra, entre $100 \mathrm{~m}^{2}$ y 1.8 ha (Ibisch, 1996; Londoño \& Álvarez, 1997). Ambos análisis se hicieron con el programa Estimates v.9.1.0 (Colwell, 2013).

Para entender de qué manera la reducción en el tamaño mínimo de los individuos incluidos en la muestra afecta los resultados, se calcularon la riqueza y la abundancia relativas (\% con respecto al total), por clases diamétricas de dos centímetros de amplitud, en un rango entre $0 \mathrm{~cm}$ y el diámetro máximo del tronco $(40 \mathrm{~cm})$ encontrado en la parcela. Posteriormente, se 
ajustó un modelo no lineal de la forma $\mathrm{Y}=$ $\mathrm{a}^{*}(\mathrm{D})^{-\mathrm{b}}$ (donde $\mathrm{Y}=$ riqueza o abundancia relativa, (D) $=$ marca de clase, a y $b=$ coeficientes del modelo), para ilustrar la tendencia de la riqueza de especies con respecto a la variación en el tamaño de los individuos considerado en la muestra. Adicionalmente, se presenta la riqueza de especies para los diferentes hábitos en diferentes categorías de tamaño, para la que se consideró diferentes áreas de muestreo $(0.1$, 0.25 y 1 ha), para facilitar la comparación con otros estudios similares.

Para analizar las diferencias entre los resultados de Montevivo y otros tipos de bosque en el neotrópico, se construyó una base de datos con reportes de la literatura que incluyen la RTE, la precipitación promedia anual y la temperatura de cada sitio donde se realizó el inventario (ver Apéndice digital 2); la mayor parte de esta información proviene del Material Suplementario de Linares-Palomino et al. (2009). Estos datos fueron agrupados de la siguiente manera de acuerdo con la clasificación de zonas de vida de Holdridge et al. (1971): Bosques húmedos tropicales o de tierras bajas bh-T, Bosques secos de tierras bajas bs- $\mathrm{T}$, Bosques húmedos montano bajos bh-Mb y las tres categorías de bosque definidas por Linares-Palomino et al. (2009): bosques Premontano Deciduo (PMd), semideciduo (PMsd) y siempre verde (PMsv), que corresponden en el sistema de Holdridge y Grenke (1971) al Bosque húmedo Premontano bh-Pm, pero diferenciados localmente por su posición topográfica, que condiciona la disponibilidad de agua del suelo.

\section{RESULTADOS}

Riqueza total de especies y su abundancia: En la parcela de 1 ha, se registraron 14545 individuos, distribuidos en 318 especies y morfoespecies. De estas, 253 fueron plenamente identificadas hasta especie (excicados con género y epíteto) y 65 quedaron registradas como morfoespecies (63 excicados identificados solo hasta género, 1 sólo hasta familia y 1 totalmente indeterminado). De aquí en adelante nos referiremos a las "especies" sin hacer diferencia con las "morfoespecies", como se acostumbra en este tipo de estudios (Gentry \& Dodson, 1987; Duivenvoorden, 1994; Galeano et al., 1998; Linares-Palomino et al., 2009).

Las angiospermas estuvieron distribuidas en 71 familias y 148 géneros; para las Pteridophytas se encontraron 49 especies y 23 géneros. El listado completo de las especies / morfoespecies en la parcela se puede consultar en David-Higuita y Álvarez-Dávila (2015). La figura 1A muestra la curva especies - área, observada y estimada con Chao 2 para familias, géneros y especies para toda la muestra; estos gráficos indican la posibilidad de registrar un mayor número de especies. De acuerdo con el modelo Chao 2, el número máximo esperado para la parcela en Montevivo fue de 413 especies, 190 géneros y 77 familias. Con base en lo anterior, la riqueza observada de familias, géneros y especies equivale al $92 \%, 90 \%$ y $77 \%$ respectivamente de la esperada.

Riqueza de especies por hábito de crecimiento: Para la muestra total de una ha, los arbustos contienen la mayor riqueza de especies (26\% del total), seguido de los árboles $(19 \%)$, las epífitas y las hierbas terrestres (ambos con $18 \%$ del total de especies), las enredaderas (11\% del total) y las lianas $(8 \%$ del total) (Cuadro 1). Este mismo orden se mantuvo para la riqueza de especies, cuando se consideraron parcelas más pequeñas de 0.25 ha y 0.1 ha. En total, los grupos no arbóreos (hierbas, escandentes, epífitas y arbustos) representaron el $81 \%$ del total de especies. El mayor número de individuos correspondió a los árboles y le siguen los arbustos.

Riqueza de especies en relación con el tamaño de los individuos: La figura $1 \mathrm{~B}$ muestra cómo la riqueza de especies aumenta exponencialmente, a medida que se disminuye el diámetro mínimo considerado en el inventario. El $95 \%$ y $92 \%$ de las especies fueron registradas por debajo de $2.5 \mathrm{~cm}$ y $1 \mathrm{~cm}$ de (D), respectivamente. Las especies encontradas por encima de los $10 \mathrm{~cm}$ equivalen solo al 16 
$\%$ del total y por encima de $5 \mathrm{~cm}$ al $24 \%$ del total (Cuadro 1).

\section{La parcela de Montevivo en un contexto} neotropical: Existen muy pocos estudios comparables con el presente, los cuales se resumen en la figura 2 y en el Apéndice digital 2. Las curvas indican que la reserva Montevivo tiene una riqueza total observada (Mv raref) de plantas vasculares superior a los reportes para bosques Premontanos deciduos (PMd) y superior a los Montanos húmedos (Mh) del neotrópico, pero inferior a los bosques tropicales de tierras bajas (TBh) y a los bosques premontanos azonales (PMsd y PMsv) (Fig. 2). En el caso de la riqueza estimada (Mv Chao 2 en la Fig. 2), esta solo es superada por la de los bosques húmedos de tierras bajas.

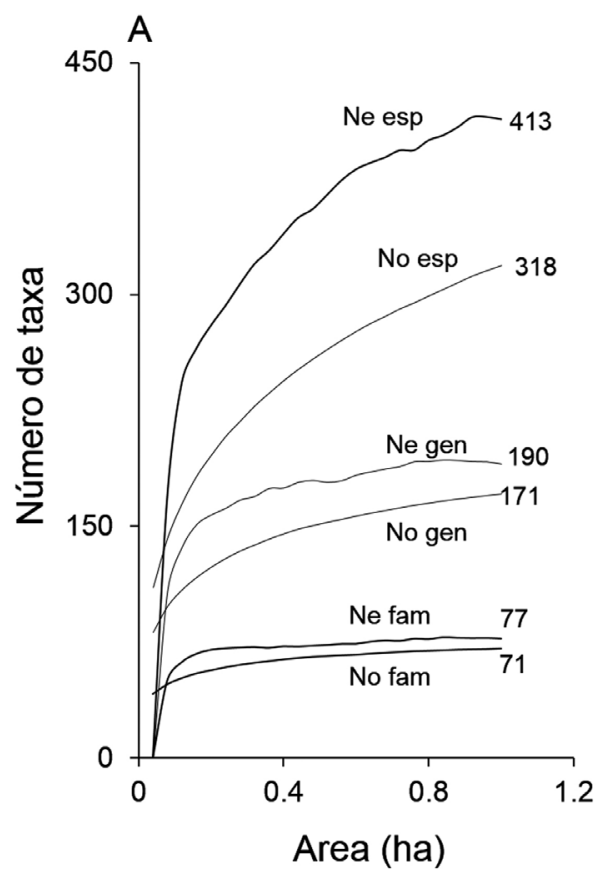

\section{DISCUSIÓN}

En este estudio se encontraron 318 especies de plantas vasculares en una hectárea de bosque alto andino. De acuerdo con DavidHiguita y Álvarez-Dávila (2015), esta riqueza representa el $47 \%$ de las especies reportadas para la flórula del Parque Arví, una reserva natural de 11400 ha aledaña al sitio donde se realizó el presente estudio, lo que demuestra la importancia de considerar todos los hábitos de crecimiento y tamaños de los individuos en los inventarios de diversidad florística. Aún más, teniendo en cuenta que solo se efectuó el conteo total en una fracción $(0.25 \mathrm{ha})$ de la parcela de una hectárea, es posible esperar que el número de especies, géneros y familias aumente como lo indica el estimador Chao 2. La riqueza de especies decrece exponencialmente

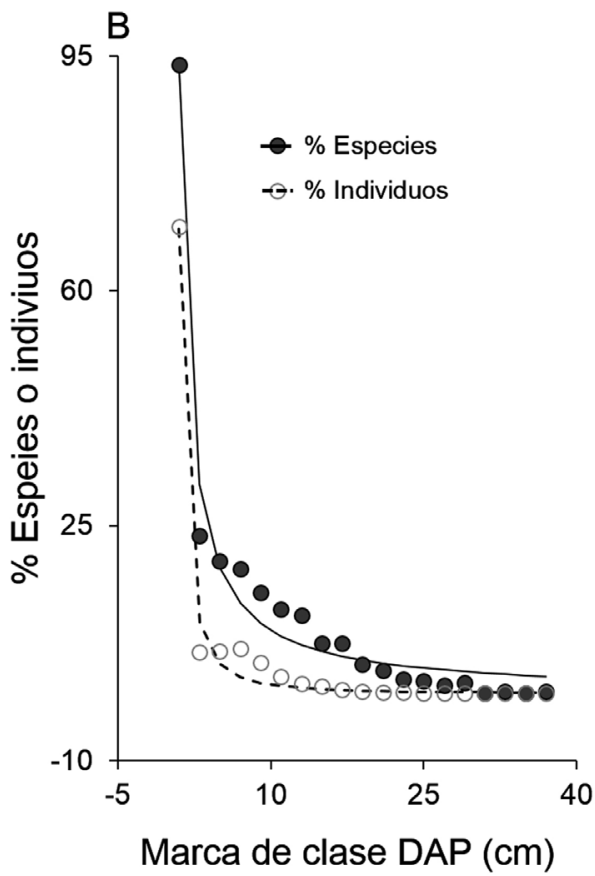

Fig. 1. A) Curvas de acumulación de especies, géneros y familias en este estudio; observadas (No) y estimadas con el modelo Chao2 (Ne). B) Relación entre el incremento en el (D) y la riqueza de especies de plantas vasculares (círculos negros y línea punteada) y el número de individuos (círculos blancos y línea continua). Se presentan los valores en \% para cada clase diametrica con respecto al total y la curva de tendencia.

Fig. 1. A) Richnnes accumulation curves for species, genera and families in this study; observed (No) and estimated with Chao2 model (Ne). B) Relation between the increase in (D) and richness of vascular plants species (black circles, dotted line) and individuals density (white circles, continuos line). Values as $\%$ for each size class are presented with respect to the total. 
CUADRO 1

Distribución por categorías de tamaño $(\mathrm{Ct})$ y hábitos de crecimiento del número total de especies, registrado en una parcela de una hectárea en este estudio

TABLE 1

Size categories $(\mathrm{Ct})$ and growth form distribution of the total number of species recorded at one hectare plot in this study

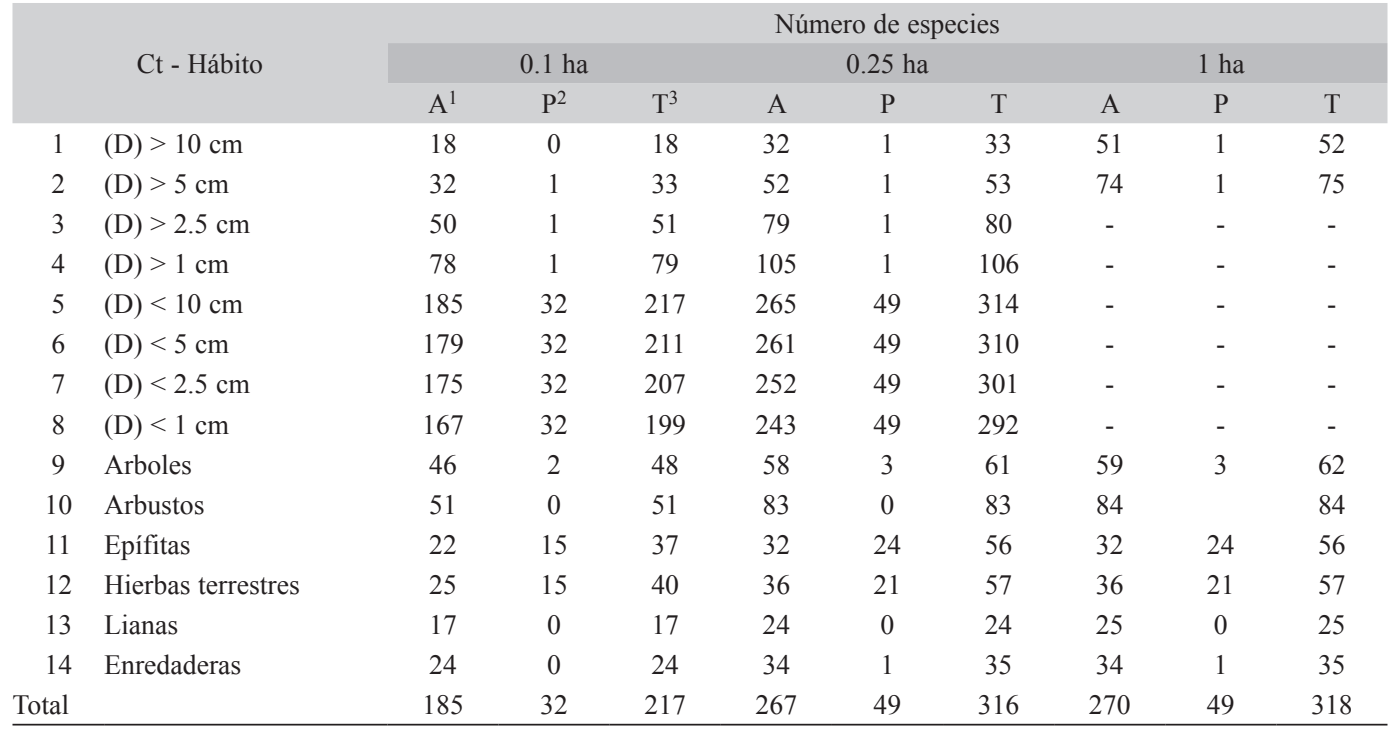

1. A: Angiosperma/Angiosperm.

2. P: Pteridophyta/Pteridophyta.

3. $\mathrm{T}$ : Total $=$ Angiosperma + Pteridophyta $/$ Angiosperm + Pteridophyta .

a medida que aumenta el diámetro mínimo de los individuos en el inventario; por ejemplo, las especies de plantas leñosas con (D) $\geq 2.5$ y (D) $\geq 10 \mathrm{~cm}$, contribuyen solamente con el $16 \%$ y $25 \%$, respectivamente, a la riqueza total. Este es un patrón que se repite en los pocos estudios disponibles que consideran todas las plantas vasculares en el muestreo (Linares-Palomino et al., 2009) y evidencia un gran vacío de conocimiento, ya que una gran proporción de los estudios más detallados realizados en los bosques tropicales, se refieren sólo al componente leñoso con (D) $\geq 2.5 \mathrm{~cm}$ (Phillips \& Miller, 2003).

Según la información que manejamos, sólo existen otros dos estudios en el neotrópico que incluyen inventarios de todas las plantas vasculares en parcelas de 1 ha; uno en un bosque húmedo de tierras bajas en Cuyabeno, Amazonia Ecuatoriana (Balslev et al., 1998) y otro en un bosque estacional a $1000 \mathrm{msnm}$ en la región de los Volcanes, en Bolivia (Linares-Palomino et al., 2009). En Cuyabeno se encontraron 942 especies de plantas vasculares en 88 familias, mientras que en Los Volcanes se encontró un promedio de $353 \pm 49$ (rango 297-382 especies / ha) con base en tres parcelas. En comparación con Cuyabeno y la parcela con mayor riqueza en los Volcanes, la riqueza de Montevivo es mucho más baja; no obstante, al considerar que en nuestro estudio el conteo total se hizo con base en un muestreo y no en la totalidad de la parcela de una ha, podemos suponer que la riqueza de Montevivo es similar a la más alta de los volcanes; esta suposición es respaldada por las 413 especies estimadas con Chao 2 en el presente estudio. Otra diferencia entre nuestro estudio y los bosques mencionados, radica en la proporción de especies por hábito de crecimiento; en Cuyabeno y los Volcanes, los arboles fueron el grupo más importante (con un 


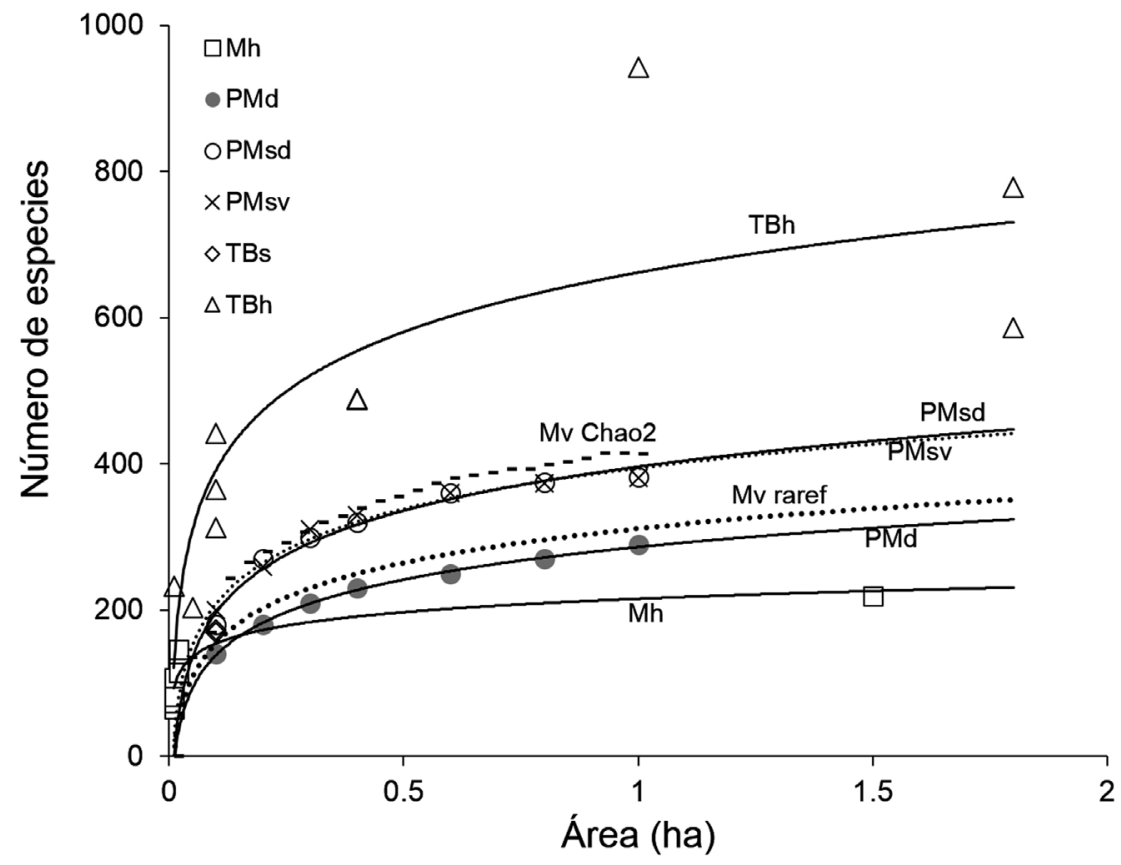

Fig. 2. Curvas de rarefacción de especies para los resultados en este estudio (Mv Chao2 y Mv raref) comparadas con curvas especies-área ajustadas con un modelo logarítmico para los datos de otros estudios en el neotrópico. Los datos se presentan agrupados por Zona de Vida: Mh: Montano húmedo, PMd: Premontano deciduo, PMsd; Premontano semideciduo, PM: premontano siempre verde, TBs: Tierras bajas seco, TBh: Tierras bajas húmedo. Ver Apéndice digital 2 para detalles.

Fig. 2. Species rarefaction curves in this study ( $\mathrm{Mv}$ Chao2 and Mv raref) compared to species-area curves adjusted with a logarithmic model for data from other studies in the neotropics. Data are presented grouped by Life Zone. Mh: Montane wet, PMd: Premontane deciduous, PMsd; Premontane semi-deciduous, PM: Premontane evergreeen, TBs: Lowland dry, TBh: Lowland wet. See appendix 2 for details.

$50 \%$ y un $29.5 \%$ respectivamente) seguido de las epífitas (18\% y $25.5 \%$, respectivamente), mientras que en Montevivo el hábito dominante correspondió a los arbustos (26.4\%), seguido de los arboles (19.2\%), los escandentes (18.9\%) y las epífitas y hierbas terrestres con un contribución bastante similar (17.6 - 17.9\%, respectivamente). Al comparar la riqueza de Montevivo con otros reportes agrupados por tipos de bosque, encontramos que los bosques húmedos de tierras bajas (TBh) tienen consistentemente un mayor número de especies plantas vasculares, mientras que los bosques montanos húmedos $(\mathrm{Mh})$, con un máximo de 219 especies / 1.5 ha (Kelly, Tanner, Lughadha, $\&$ Kapos, 1994), tienen una menor riqueza.
Otros estudios comparables en parcelas con área mayor a 0.4 ha, reportan que los árboles de todos los tamaños representan el $25.6 \%$ y $31.7 \%$ de la RTE en bosques del Choco (Galeano et al., 1998), el $60 \%$ en un bosque húmedo de la Amazonia colombiana (Londoño \& Álvarez 1997), y el 18.8 \% en un bosque montano húmedo en Venezuela (Kelly et al., 1994); este último valor es similar al encontrado en Montevivo. La mayor cantidad de reportes sobre riqueza de plantas escandentes se refiere a bosques tropicales por debajo de los $1000 \mathrm{msnm}$; por ejemplo, Gentry y Dodson (1987) muestran que la contribución de este grupo a la riqueza total varía entre 13 y $37 \%$ para diferentes regiones, y varía en relación con 
un gradiente de precipitación. En la Amazonia Ecuatoriana, Balslev et al. (1998) encontraron 99 especies de plantas escandentes en 0.49 ha (10\% del total), mientras que Duivenvoorden (1994) encontró una riqueza equivalente al 7 $\%$ del total. Otro estudio en tierras bajas súper húmedas del Chocó Biogeográfico en Colombia, encontró que las lianas representan entre el $4.1-8.8 \%$ del total de especies en parcelas de 0.4 ha (Galeano et al., 1998). Linares-Palomino et al. (2009) reportaron entre 44 y 86 especies escandentes/ha a los $1000 \mathrm{msnm}$ en un gradiente de humedad del suelo en los andes Bolivianos, que representaron el $22 \%$ del total, en los sitios con déficit de humedad y apenas el 11 $\%$ en el bosque húmedo; en el presente estudio, el $18.9 \%$ de las especies son escandentes, lo que muestra que el grupo es importante también en los bosques andinos. Las 57 especies de hierbas terrestres que representan el $15.7 \%$ del total; este grupo contribuye entre un 10-14\% para los bosques de los andes Bolivianos donde se reportan entre 30 - 57 especies/ha (LinaresPalomino et al., 2009), $14 \%$ en el Chocó colombiano con 63 especies/0.1 ha (Galeano et al., 1998), entre 18-50 especies en 0.1 ha en los bosques húmedos y deciduos de tierras bajas en Ecuador representando entre 10.7 y $28.9 \%$ del total (Gentry \& Dodson, 1987). Por último, $17.6 \%$ de las especies en nuestro estudio son epífitas; una proporción similar a la reportada para Cuyabeno (18\% en Balslev et al., 1998), y menor que los demás estudios consultados: $20 \%$ a $28 \%$ para los andes Bolivianos (Linares-Palomino et al., 2009), 25 \% para el Chocó (Galeano et al., 1998), $35 \%$ en el Occidente del Ecuador (Gentry \& Dodson, 1987) y $25 \%$ para Costa Rica (Hammel, 1990).

Nuestros resultados muestran la alta riqueza de especies que puede contener una hectárea de bosque andino cuando se consideran todas las plantas vasculares. Los árboles con (D) $\geq 10 \mathrm{~cm}$, y las plantas leñosas con $(\mathrm{D}) \geq 2.5$ $\mathrm{cm}$, que representan los tamaños mínimos más reportados en la literatura de inventarios florísticos, representan una proporción menor de la RTE en la parcela estudiada, similar a lo reportado en los estudios consultados (con promedios de $19.1 \pm 7.7 \%$ y $39.7 \pm 14.7 \%$, respectivamente). Por su parte, la contribución promedio de los hábitos de crecimiento no arbóreos (escandentes, hierbas, epífitas, sin incluir arbustos) a la RTE es de 44.8 $\pm 31.5 \%$ (con base en los estudios publicados) un rango en el que se encuentra el valor de Montevivo donde estos hábitos de crecimiento representan el $54.3 \%$, confirmando que son elementos importantes en la estructura, composición y riqueza de especies de los bosques andinos. En conclusión, este estudio y los demás publicados con información sobre la RTE, confirman que los inventarios florísticos en parcelas generalmente ignoran más de la mitad de las especies presentes en un sitio determinado. Por lo tanto, para estudios futuros donde se requiera evaluar la diversidad de plantas para los objetivos de conservación, se recomienda considerar todas las formas de crecimiento y no solo los árboles grandes o las plantas leñosas; de esta manera se tendrá una idea más precisa sobre lo que se espera conservar y así tomar mejores decisiones para la planificación y manejo de áreas protegidas.

Un asunto importante adicional, tiene relación con los patrones de distribución espacial de la RTE y sus determinantes ambientales. Aunque en años recientes ha aumentado el conocimiento sobre los patrones de riqueza de plantas en grandes escalas usando parcelas y grupos indicadores como el de los árboles grandes (Ter Steege et al., 2013), plantas leñosas (Phillips \& Miller, 2003) o epífitas (Kuper, Kreft, Nieder, Koster, \& Barthlott, 2004), los patrones de la RTE son desconocidos. De acuerdo con nuestra revisión, solo existen una docena de estudios con objetivos de cuantificar la RTE en el neotrópico, con el inconveniente adicional de que utilizan diferentes áreas de muestreo. Sin embargo, los resultados de estos estudios sugieren algunos patrones similares a los reportados para árboles y plantas leñosas, con una riqueza máxima en los bosques húmedos de tierras bajas, que decrece simultáneamente hacia los sitios con menor precipitación y menor temperatura. Adicionalmente, en los estudios revisados, se encuentran evidencias de 
que la topografía y la variación asociada con las condiciones del suelo, determinan diferentes tipos de vegetación en sitios con condiciones climáticas homogéneas en los Andes (Jarvis \& Mulligan, 2001). Por ejemplo, el estudio de Linares-Palomino et al. (2009), muestra los efectos a escala local, del suelo y la orientación de las parcelas en la diversidad de los bosques andinos; su área de estudio tiene condiciones similares de precipitación y temperatura, que determinan la presencia zonal de un "bosque Semideciduo" (alrededor de 30 - 50 \% árboles de hoja caduca) predominando en las laderas con orientación sur con baja incidencia directa de la radiación solar; adicionalmente, en las laderas con orientación norte que tienen mayor incidencia de radiación solar, se encuentran "bosques caducifolios" (70 - 90 \% árboles de hoja caduca), mientras que en los valles planos, con sombra, y buen suministro de agua subterránea se encuentran bosques de hoja perenne (10 - $20 \%$ árboles de hoja caduca). A pesar de que la altitud es un buen indicador de la dinámica del agua-energía en los sistemas de montaña (O'Brien, Field, \& Whittaker, 2000), el estudio de Linares-Palomino et al. (2009) confirma que el relieve es un determinante importante de la heterogeneidad florística de los bosques andinos (Jentsch \& Beierkuhnlein, 2003).

\section{AGRADECIMIENTOS}

Este proyecto fue financiado con fondos personales de los autores, y hace parte de los productos de la Red Col-Tree para el monitoreo de los bosques de Colombia. Especial agradecimiento a Dario Cock, por facilitar la reserva para el montaje de la parcela y por la logística prestada. A los biólogos y biólogas quienes colaboraron en la fase de campo. A los especialistas que colaboraron en la determinación del material botánico: R. Callejas, F. J. Roldan, J. Betancur, F. Cardona, F. Alzate, W. Rodriguez, F. Giraldo, J. Perez. A Lina Vélez por su colaboración en la base de datos, y a Wilson Rengifo por su colaboración en el taller de HUA. Reynaldo Linares-Palomino facilitó el acceso a sus datos de RTE. Para la elaboración del artículo Esteban Álvarez recibió apoyo de Colciencias, la Universidad de Leeds, La Fundación Convida Medellín y la Universidad Nacional abierta y a Distancia.

\section{RESUMEN}

Los reportes sobre diversidad de plantas en los bosques tropicales suelen estar restringidos a árboles u otros grupos de plantas leñosas por encima de cierto diámetro del tallo. Sin embargo, otros estudios que incluyen todas las formas de vida sin restricciones de tamaño de los individuos, indican claramente que las plantas no leñosas son igual de importantes. En este estudio se reporta la Riqueza total de especies de plantas vasculares (RTE) en una parcela de una hectárea en un bosque andino del noroccidente de Colombia ( $6^{\circ} 12^{\prime} 48^{\prime \prime} \mathrm{N} \& 7^{\circ} 29^{\prime} 32^{\prime}$ 'O); adicionalmente evaluamos la contribución de los diferentes hábitos de crecimiento a la RTE y el efecto del tamaño de las plantas. Se censaron todos los individuos con diámetro del tronco (D) $\geq 5 \mathrm{~cm}$ en 1 ha, y todas las plantas vasculares de todos los tamaños, incluyendo epífitas, en una muestra de 0.25 hectáreas. Se registró un total de 14545 individuos distribuidos en 318 especies, 72 familias (considerando Pteridophyta como un solo grupo) y 171 géneros. El 99.7 $\%$ de las especies son menores de $10 \mathrm{~cm}$ de (D) y el 94.4 $\%$ son menores a $2.5 \mathrm{~cm}$ de (D). Las especies no arbóreas (hierbas terrestres, epífitas y escandentes) representan el $54.3 \%$ del total de especies registradas en la parcela, lo que indica que son un componente clave de la estructura, composición y riqueza de este bosque montano neotropical. Estos resultados confirman reportes similares para otros bosques tropicales. Concluimos que para conocer con más detalle la diversidad de florística de un sitio es recomendable: 1) ampliar el rango de tamaño de las plantas considerado comúnmente en los inventarios florísticos y 2) incluir las especies no leñosas; esta información es crucial para tomar mejores decisiones en los esfuerzos de conservación a escala local y global.

Palabras clave: diversidad florística; conteo total; hábitos de crecimiento; especies no leñosas; rangos de medición.

\section{REFERENCIAS}

Balslev, H., Valencia, R., Paz y Miño, G., Christensen, H., $\&$ Nielsen, I. (1998). Species count of vascular plants in one hectare of humid lowland forest in Amazonian Ecuador. In J. A. Comiskey \& F. Dallmeier (Eds.), Forest biodiversity in North, Central and South America, and the Caribbean: research and monitoring (pp. 585-594). Paris, France: The Parthenon Pub. Group.

Benavides, A. M., Vasco, A., Duque, A. J., \& Duivenvoorden, J. F. (2011). Association of vascular epiphytes 
with landscape units and phorophytes in humid lowland forests of Colombian Amazonia. Journal of Tropical Ecology, 27, 223-237. doi:10.1017/ S0266467410000726

Bordenave, B. G., Granville, J. De, \& Hoffman, M. (1998). Measurement of species richness of vascular plants in a neotropical rain forest in French Guiana. In J. A. Comiskey \& F. Dallmeier, (Eds.), Forest biodiversity in North, Central and South America, and the Caribbean: research and monitoring (pp. 411-425). Paris, France: The Parthenon Pub. Group.

Chao, A., Colwell, R. K., Lin, C. W., \& Gotelli, N. J. (2009). Sufficient sampling for asymptotic minimum species richness estimators. Ecology, 90(4), 1125-1133.

Colwell, R. K. (2013). EstimateS: Statistical estimation of species richness and shared species from samples. Version 9. User's Guide and application. Retrieved from http://doi.org/10.1613/jair.301

Colwell, R. K., Chao, A., Gotelli, N. J., Lin, S. Y., Mao, C. X., Chazdon, R. L., \& Longino, J. T. (2012). Models and estimators linking individual-based and samplebased rarefaction, extrapolation and comparison of assemblages. Journal of Plant Ecology, 5(1), 3-21. doi:10.1093/jpe/rtr044

David-Higuita, H., \& Álvarez-Dávila, E. (2015). Representatividad a escala regional de un inventario florístico detallado de una hectárea en los andes tropicales. Colombia Forestal, 18(2), 207-224. doi:10.14483/ udistrital.jour.colomb.for.2015.2.a03

Duivenvoorden, J. F. (1994). Vascular plant species counts in the rain forests of the middle Caqueta area, Colombian Amazonia. Biodiversity and Conservation, 3, 685-715. doi:10.1007/BF00126860

Galeano, G., Suárez, S., \& Balslev, H. (1998). Vascular plant species count in a wet forest in the Choco area on the Pacific coast of Colombia. Biodiversity \& Conservation, 7, 1563-1575. doi:10.1023/A:1008802624275

Gentry, A. H. (1995). Patterns of diversity and floristic composition in Neotropical montane forests. In Churchill, S. P., Balslev, H., Forero, E., \& Luteyn, J. L. (Eds.). Proceedings of a symposium, New York Botanical Garden Biodiversity and Conservation of Neotropical Montane Forests (pp. 103-126). New York: New York Botanical Garden.

Gentry, A. H., \& Dodson, C. (1987). Contribution of nontrees to species richness of a tropical rain forest. Biotropica, 19(2), 149-156. doi:10.2307/2388737
Hammel, B. (1990) The distribution of diversity among families, genera, and habit types in the La Selva Flora. In A. H.Gentry (Ed.), Four neotropical rainforests (pp. 75-84). New Haven and London: Yale University Press.

Holdridge, L. R., \& Grenke, W. C. (1971). Forest environments in tropical life zones: a pilot study. Forest environments in tropical life zones: a pilot study.

Holdridge, L. R., Grenke, W., Hatheway, W. H., Liang, T., Tosi, J. A. (1971). Forest Environments in Tropical Life Zones: A Pilot Study. Oxford: Pergamon Press.

Ibisch, P. L. (1996). Neotropische Epiphytendiversitat: das Beispiel Bolivien. Archiv naturwissenschaftlicher Dissertationen, 1, 1-357.

Jarvis, A., \& Mulligan, M. (2011). The climate of cloud forests. Hydrological Processes, 25(3), 327-343.

Jentsch, A., \& Beierkuhnlein, C. (2003). Global Climate Change and Local Disturbance Regimes as Interacting Drivers for Shifting Altitudinal Vegetation Patterns. Erdkunde, 216-231.

Kelly, D., Tanner, E., Lughadha, E., \& Kapos, V. (1994). Floristics and biogeography of a rain forest in the Venezuelan Andes. Journal of Biogeography, 21(4), 421-440.

Kuper, W., Kreft, H., Nieder, J., Koster, N., \& Barthlott, W. (2004). Large-scale diversity patterns of vascular epiphytes in Neotropical montane rain forests. Journal of Biogeography, 31, 1477-1487.

Linares-Palomino, R., Cardona, V., Hennig, E. I., Hensen, I., Hoffmann, D., Lendzion, J., ... Kessler, M. (2009). Non-woody life-form contribution to vascular plant species richness in a tropical American forest. Plant Ecology, 2001(1), 87-99. doi:10.1007/978-90-481-2795-5_8

Londoño, A. C., \& Álvarez, E. (1997). Composición florística de dos bosques (tierra firme y varzea) en la región de Araracuara, Amazonia colombiana. Caldasia, 19(3), 431-463.

Mora, C., Tittensor, D., Adl, S., Simpson, A., \& Worm, B. (2011). How many species are there on Earth and in the ocean? PLoS Biol, 9(8), e1001127. doi:10.1371/ journal.pbio. 1001127

O'Brien, E. M., Field, R., \& Whittaker, R. J. (2000). Climatic gradients in woody plant (tree and shrub) diversity: water-energy dynamics, residual variation, and topography. Oikos, 89(3), 588-600. 
Oldeman, R. A. A. (1990). Forests: elements of silvology. Berlin: Springer-Verlag.

Perry, D. R., (1978). A method of access into the crowns of emergent and canopy trees. Biotropica, 10, 155-157.

Phillips, O. L., \& Miller, J. . S. (2003). Global patterns of plant diversity. In H. Alwyn (Ed.), Gentry's Forest Transect Data Set. USA: Economic Botany.

Phillips, O. L., Vásquez-Martínez, R., Núñez-Vargas, P., Monteagudo, A. L., Chuspe-Zans, M., GalianoSánchez, W., ... Rose, S. (2003). Efficient plotbased floristic assessment of tropical forests. Journal of Tropical Ecology, 19(6), 629-645. doi:10.1017/ S0266467403006035

Prance, G. T., Beentje, H., Dransfield, J., \& Johns, R. (2000). The tropical flora remains undercollected. Annals of the Missouri Botanical Garden, 87, 67-71.
Qian, H., \& Song, J. (2013). Latitudinal gradients of associations between beta and gamma diversity of trees in forest communities in the New World. Journal of Plant Ecology, 6(1), 12-18.

Scheiner, S. M. (2003). Six types of species-area curves. Global ecology and biogeography, 12(6), 441-447. doi:10.1093/jpe/rts040

ter Steege, H., Pitman, N. C. A., Sabatier, D., Baraloto, C., Salomão, R. P., Guevara, J. E., ... Ree, R. H. (2013). Hyperdominance in the Amazonian tree flora. Science, 342(6156), 1243092. doi:10.1126/ science. 1243092

Whitmore, T. C., Peralta, R., \& Brown, K. (1985). Total species count in a Costa Rican tropical rain forest. Journal of Tropical Ecology, 1(4), 375-378. doi:10.1017/S0266467400000481 